\title{
Increased Choroidal Thickness in Morquio Syndrome
}

\author{
Augusto Magalhães ${ }^{a}$ Rodrigo Vilares-Morgado ${ }^{a, b}$ \\ Ana Maria Cunha ${ }^{a}$ Elisa Leão-Teles ${ }^{c}$ Manuel Falcão ${ }^{a, b}$ \\ Ângela Carneiro ${ }^{a, b}$ Fernando Falcão-Reis ${ }^{a, b}$ \\ aDepartment of Ophthalmology, Centro Hospitalar Universitário de São João, Porto, \\ Portugal; ${ }^{b}$ Department of Surgery and Physiology, Faculty of Medicine of University of

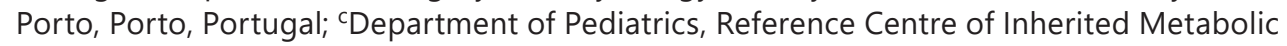 \\ Diseases, Centro Hospitalar Universitário de São João, Porto, Portugal
}

\section{Keywords}

Morquio syndrome $\cdot$ Choroidal thickness - Optical coherence tomography

\begin{abstract}
The purpose of this clinical case report is to describe a case of mucopolysaccharidosis type IVA (MPS IVA), or Morquio syndrome, with increased choroidal thickness in enhanced-depth imaging optical coherence tomography (EDI-OCT) which can represent choroidal deposition of glycosaminoglycans (GAGs). A 21-year-old male with genetically confirmed diagnosis of MPS IVA was examined at our Pediatric Ophthalmology clinic as part of our follow-up protocol for MPS patients. His best-corrected visual acuity was $4 / 10$ in his right eye (OD) and 6/10 in the left eye (OS). Mild diffuse corneal opacification was evident. Intraocular pressure was within normal range. Fundus examination and color fundus photography revealed no abnormalities. EDI-OCT revealed significantly increased choroidal thickness in his right eye and in his left eye, suggesting the presence of choroidal deposition of GAGs, despite absence of retinal or optic disc GAG deposition or other chorioretinal involvement. To our knowledge, this is the first case of MPS IVA described in the literature with suspected choroidal deposition of GAGs. With improved control of systemic features of MPS IVA, life expectancy of these patients has increased, allowing for more ocular manifestations to develop. The parallel development of technology in ophthalmology, such as the EDI-OCT, further contributes to the detection of these unprecedented ocular features in MPSs.
\end{abstract}

\section{Karger $\stackrel{\text { ! }}{=}$}




\section{Introduction}

Mucopolysaccharidoses (MPSs) are a group of heterogeneous metabolic disorders caused by inherited defects in lysosomal enzymes resulting in systemic intra- and extracellular accumulation of glycosaminoglycans (GAGs) [1,2]. MPS type IV (MPS IV), or Morquio syndrome, includes 2 different subtypes, $\mathrm{A}$ and $\mathrm{B}$, both inherited in an autosomal recessive manner, though the gene product involved is distinct. MPS IVA, the most common form, results from a defect in the GALNS gene in chromosomal region 16q24, leading to abnormal production of $\mathrm{N}$-acetylgalactosamine-6-sulfatase, which is required for the degradation of the GAGs, keratan sulfate (KS), and chondroitin-6-sulfate [3]. Clinical features include the typical coarse facial appearance, skeletal dysplasia, short stature, cervical instability, sensorineural hearing loss, heart valvular disease, and respiratory tract malformations [4]. Nevertheless, intelligence is frequently normal or near normal [5]. Life expectancy is variable but has increased in the last 2 decades due to new therapeutic options, such as heterologous hematopoietic stem cell transplant and enzyme replacement therapy $[6,7]$.

Frequent ophthalmological features of this disease include progressive diffuse corneal cloudy infiltration due to ocular accumulation of KS and refractive errors [2]. Further reported ocular manifestations include cataracts, glaucoma, optic nerve atrophy, and optic disc swelling. Retinopathy may occur but has rarely been reported [8]. Secondary glaucoma is not as frequent as in other types of MPS [9]. Due to their shallow orbits from skeletal dysplasia, pseudoexophthalmos may occur, leading to corneal exposure keratopathy $[2,5]$.

Despite widespread ocular accumulation of GAGs, choroidal deposition has not yet been reported in MPS IVA. Thus, the aim of this case report is to describe the aforementioned feature using enhanced-depth imaging optical coherence tomography (EDI-OCT), in a patient with genetically confirmed diagnosis, as well as to discuss the pathophysiology of his ocular disease, reporting his long-term evolution.

\section{Case Presentation}

A 21-year-old Caucasian male MPS IVA patient was observed in our Pediatric Ophthalmology Department as part of our follow-up protocol. He had been diagnosed with Morquio syndrome when he was only 13 months old and had been followed-up for over 14 years in our clinic. The diagnosis had been confirmed biochemically (increased urinary GAG levels, consisting predominantly of KS; absence of galactose-6-sulfatase; and normal beta-galactosidase levels in leucocytes in fibroblasts) and genetically (c.901G > T; pG301C homozygotous mutation in exon 9 of the GALNS gene that encodes the deficient enzyme galactose-6-sulfatase). Neither family history of MPS IVA nor history of parental consanguinity had been identified (Fig. 1). He had an identical twin brother with the same disease who died at the age of 10 years. The probable cause of death was a cardiac arrhythmia.

The patient presented short stature (with a height of $110 \mathrm{~cm}$ ) and coarse facial features, including macroglossia, typical of MPSs. Fourteen years ago, he required tracheostomy and cervical decompression surgery due to acute spinal cord compression, which occurred during an episode of febrile convulsion and was related with dysplasia and hypermobility of the atlanto-occipital articulation. Since then, he has been confined to an electric wheelchair due to reduced lower limb mobility. He has also been receiving domiciliary ventilation through his tracheostomy, which includes a Passy-Muir valve to aid his speaking. He had some minor episodes of upper respiratory tract infections. Despite periodical screening echocardiograms, no cardiac dysfunction was demonstrated. His hearing was also impaired, suffering from bilateral progressive sensorineural hearing loss. Regarding his mental development, he presented a

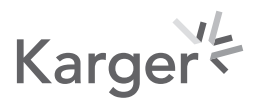




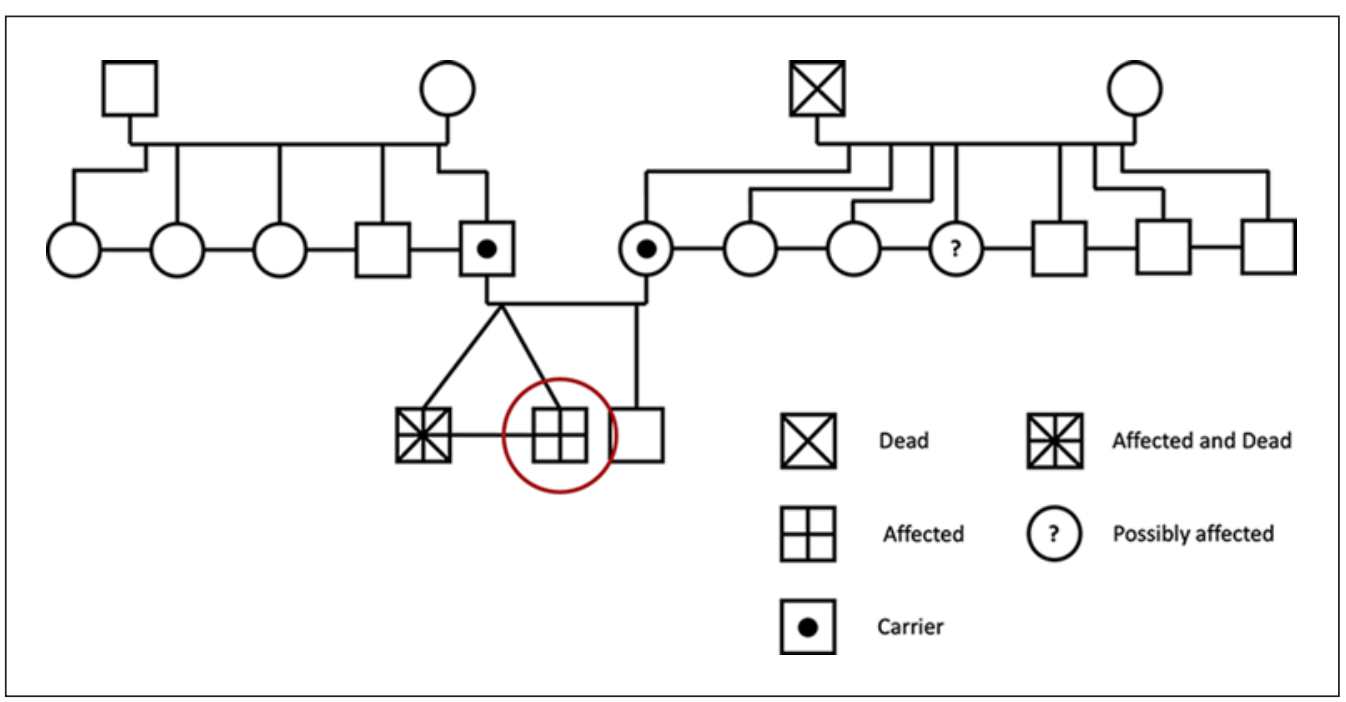

Fig. 1. Genogram of our patient (circled in red). His identical twin had the same disease and died at the age of 10 years. The probable cause of death was a cardiac arrhythmia. Both parents are disease carriers. An aunt from his mother's side may also be affected. There is no family history of consanguinity.

moderate cognitive impairment, though he was able to complete his studies in a private adapted school and ingress in higher education.

Regarding ophthalmological involvement, the patient was first evaluated at our Pediatric Ophthalmology Department at the age of 7 years, having been referred to this clinic to screen for ocular involvement of his disease. Throughout the first years of follow-up, he had a stable best-corrected visual acuity (BCVA) of 6/10 bilaterally. He required correction for his complex hyperopic astigmatism, having adapted well to spectacles. No significant corneal opacification was evident, and central corneal thickness was within normal limits. Intraocular pressure was also within normal range, with values between 14 and $18 \mathrm{~mm} \mathrm{Hg}$ bilaterally. There was no optic nerve involvement, and multimodal retinal imaging demonstrated no abnormalities.

In his most recent visit to our Pediatric Ophthalmology clinic, the patient was 21 years old and had a BCVA of 4/10 in the right eye (OD) and 6/10 in the left eye (OS) with +1.25 $+3.00 \times 80^{\circ}$ in his $\mathrm{OD}$ and $+4.00 \times 90^{\circ}$ in his OS. His corneas had mild diffuse opacification (Fig. 2). Intraocular pressure was $18 \mathrm{~mm} \mathrm{Hg}$ in OD and $16 \mathrm{~mm} \mathrm{Hg}$ in OS, having been measured with Goldman applanation tonometry. Fundus examination revealed no abnormalities. Corneal tomography with OCULUS Pentacam ${ }^{\circledR}$ revealed a central corneal thickness of $549 \mu \mathrm{m}$ in OD and $603 \mu \mathrm{m}$ in OS. We determined an axial length of $21.61 \mathrm{~mm}$ in OD and $22.03 \mathrm{~mm}$ in OS using the Carl Zeiss IOLMaster ${ }^{\circledR}$. Hardy-Rand-Rittler test revealed no color vision defects. Color fundus photography (CFP), fundus autofluorescence (FAF), and retinal EDI-OCT were then performed (Fig. 3-5). CFP and FAF revealed no pathological features. In the EDI-OCT, a subfoveal choroidal thickening of $651 \mu \mathrm{m}$ and $509 \mu \mathrm{m}$ was evident in his right and left eyes, respectively, though the retinal pigment epithelium and retinal layers had no apparent changes in both eyes, as measured with the image analysis software tool made available with the SPECTRALIS Heidelberg ${ }^{\circledR}$ HRA + OCT (Heidelberg Engineering, Heidelberg, Germany). The software tool automatically converts pixels into micrometers. Measurements were repeated 3 times, and the average of the 3 measurements was used. EDI-OCT of the optic disc revealed normal retinal nerve fiber layer thickness bilaterally. 


\section{Case Reports in Ophthalmology}
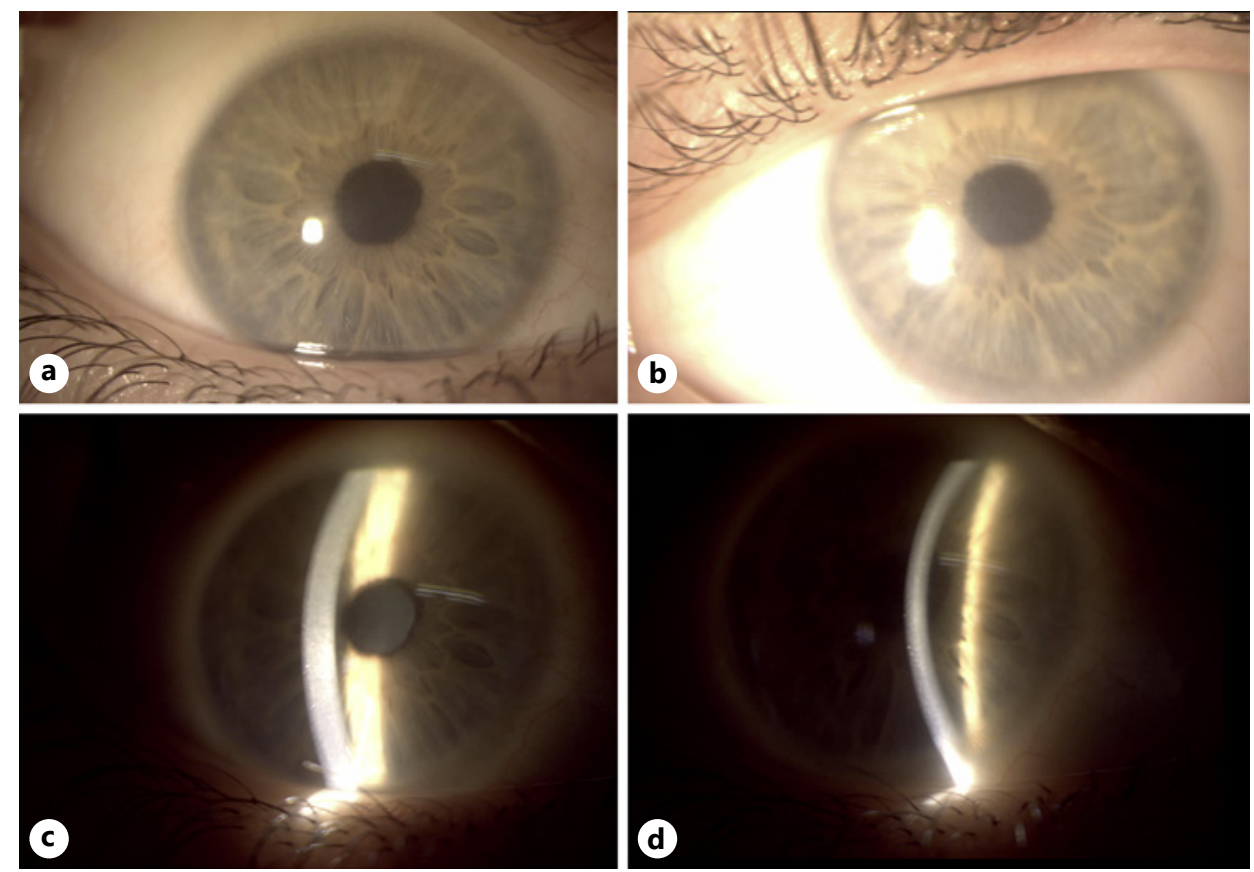

Fig. 2. Slit-lamp diffuse illumination and corneal parallelepiped photographs of the anterior segment of the right eye $(\mathbf{a}, \mathbf{c})$ and the left eye $(\mathbf{b}, \mathbf{d})$ with bilateral mild diffuse corneal opacification and bilateral mild corneal stromal infiltration.
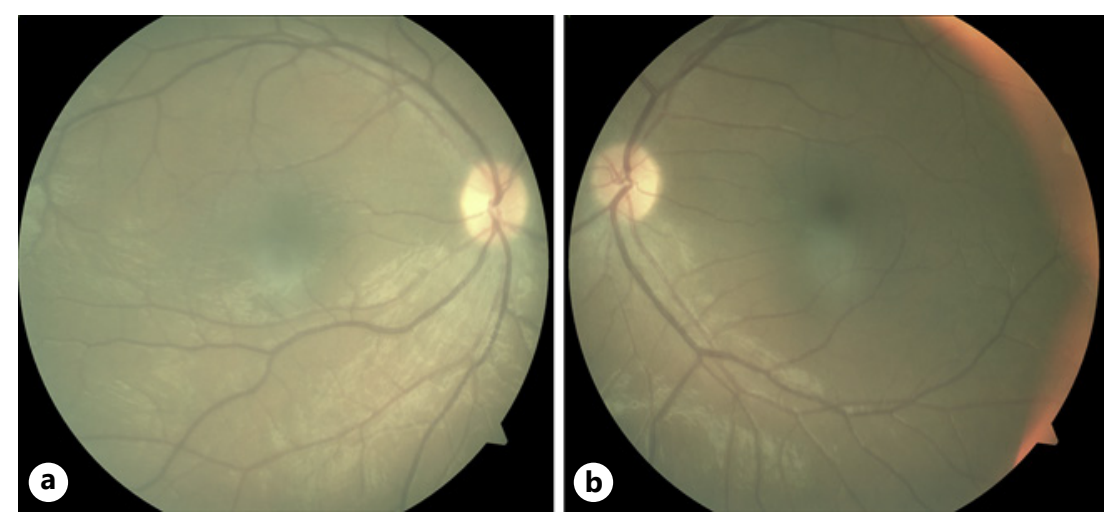

Fig. 3. Color fundus photographs of the right eye (a) and the left eye (b) revealing no apparent accumulation of glycosaminoglycans in the retina or the optic nerve.

\section{Discussion}

The most frequently described ocular manifestations of MPS IVA are progressive bilateral corneal clouding and refractive errors, which lead to decreasing visual acuity at early school age [4]. Nevertheless, corneal opacification is not as severe as in MPS I and MPS VI, and secondary glaucoma is also less common [9]. Both reasons contribute to better visual prognosis in patients with MPS IVA.

Patients with MPS IVA may present optic nerve atrophy and optic disc swelling, though our patient had a normal fundoscopic examination and normal nerve fiber layer analysis in 

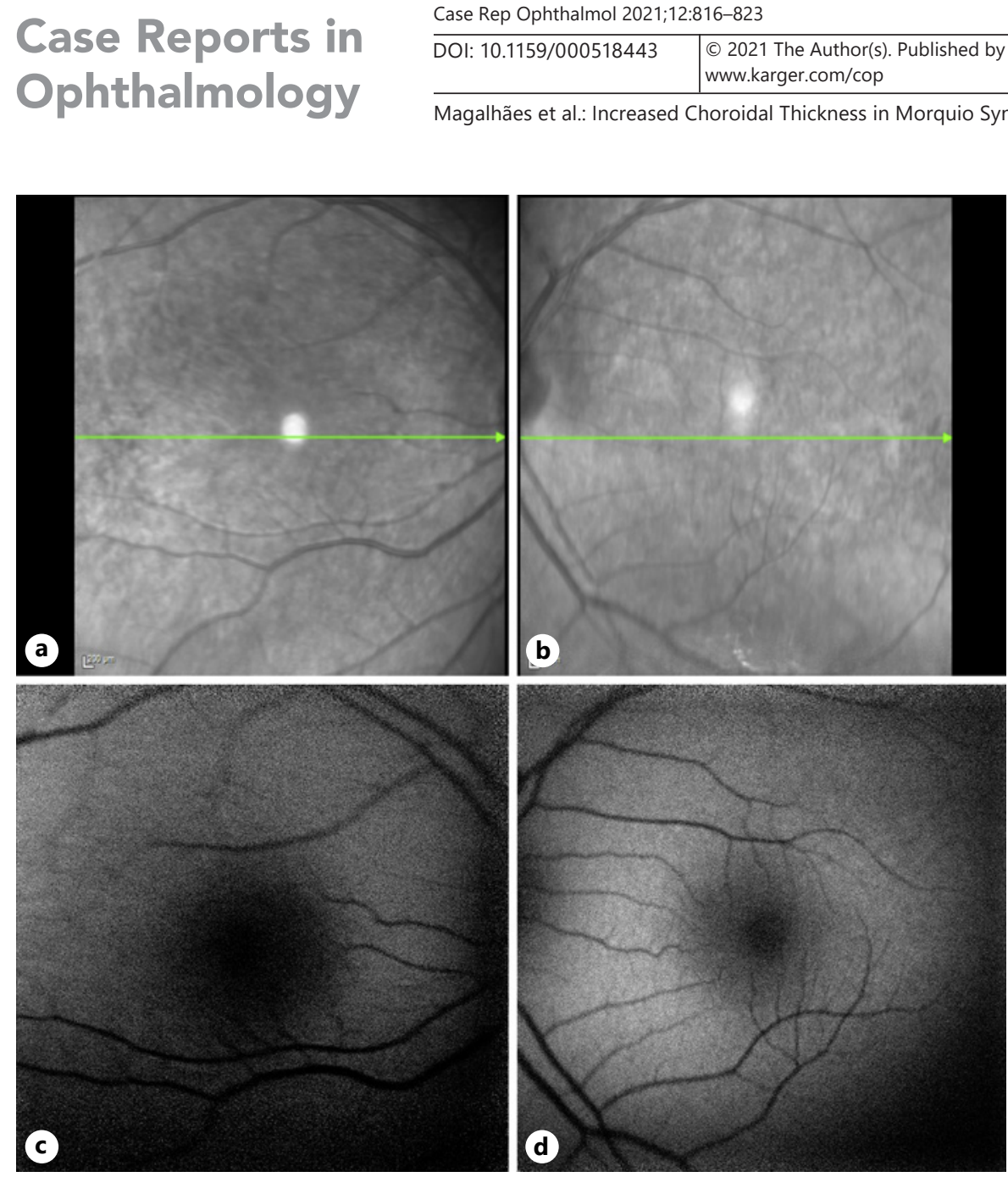

Fig. 4. Infrared fundus imaging and fundus autofluorescence imaging with SPECTRALIS Heidelberg ${ }^{\circledR} H R A+$ OCT of the right eye $(\mathbf{a}, \mathbf{c})$ and the left eye $(\mathbf{b}, \mathbf{d})$ with no apparent retinal involvement.

the OCT [2]. MPS IVA may also feature retinopathy due to retinal GAG deposition. Our patient had no retinal involvement in his fundoscopic examination, which was confirmed by multimodal retinal imaging with CFP and EDI-OCT.

Although choroidal and scleral involvement of MPSs is not frequently reported in the literature, in our Pediatric Ophthalmology Department, follow-up protocol for patients with MPSs includes monitoring this involvement, with periodic evaluation of choroidal and scleral OCT features, using more recent OCT systems, with higher image quality and better visibility of both layers. This resulted in the first case report of a mucopolysaccharidosis type VI (MPS VI) patient with fundoscopic and EDI-OCT alterations that may correspond to scleral deposition of GAGs [10]. Furthermore, a case report of a 6-year-old female patient with mucopolysaccharidosis type I (MPS I) and Hurler phenotype who presented bilateral choroidal deposition in both the CFP and in the OCT was also recently published [11]. Thus, sclerochoroidal features of MPSs are being increasingly identified using the most recent technology available.

Regarding our MPS IVA patient, we found a subfoveal choroidal thickness of $651 \mu \mathrm{m}$ in his right eye and $509 \mu \mathrm{m}$ in his left eye. Recently, Entezari et al. [12] studied choroidal thickness in normal healthy eyes using EDI-OCT. In his work, mean subfoveal choroidal thickness was $363 \pm 84 \mu \mathrm{m}$ [12]. Thus, our patient's mean subfoveal choroidal thickness of $651 \mu \mathrm{m}$ (right eye) and $509 \mu \mathrm{m}$ (left eye) can be considered significantly increased, since it falls out of a normal distribution. We hypothesized that subfoveal choroidal thick- 


\section{Case Reports in Ophthalmology}

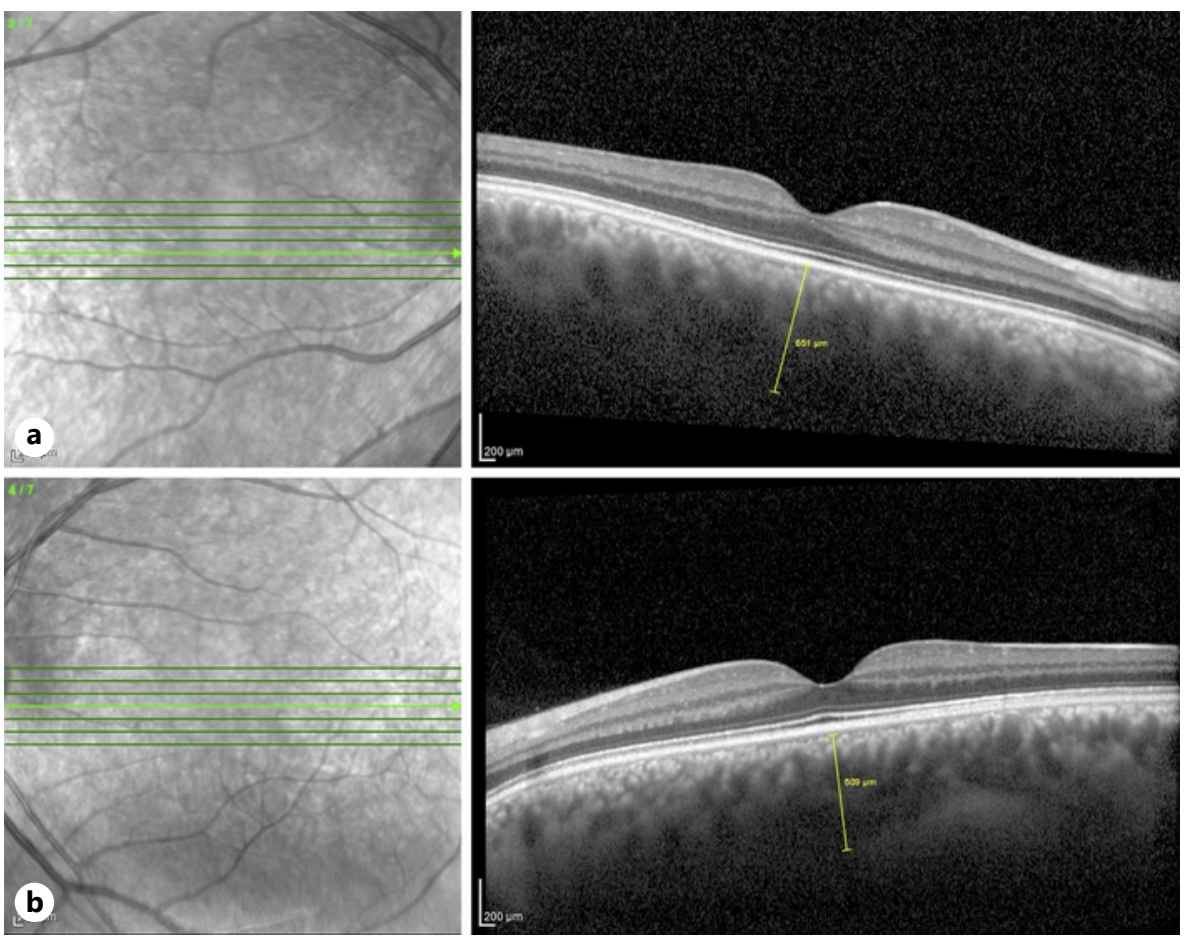

Fig. 5. Enhanced-depth imaging optical coherence tomograms of the right eye (a) and the left eye (b), revealing a subfoveal choroidal thickening of $651 \mu \mathrm{m}$ in the right eye and $509 \mu \mathrm{m}$ in the left eye, without retinal pigment epithelium or retinal layer glycosaminoglycan deposition in both eyes.

ening may result from choroidal deposition of GAGs, more specifically KS. Other causes of choroidal thickening in young adults include hyperopia [13], Graves and vascular orbitopathies [14], nephrotic syndrome, and keratoconus [15], but none of these may account for increased SFCT in this patient, since he only presented mild hyperopia with a normal axial length in both eyes and had no signs of the other pathologies. Scleral deposition of GAGs has been reported in Morquio syndrome [16], which could distort the choroidal scleral interface and lead to an overestimation of choroidal thickness, but this patient presented a regular and uniform sclera. Scleral perforating blood vessels were not present in the tomograms we obtained.

There are no similar case reports in the literature describing such features in patients with MPS IVA. This can be related to multiple reasons. First, this choroidal deposition is probably an uncommon feature of MPS IVA. Second, even when present, it may be difficult to notice since these patients often do not cooperate well enough to enable adequate resolution of captured EDI-OCT images, either due to cognitive impairment or following unstable central fixation caused by poor vision. Last, the improved control of systemic comorbidities in the last years increased the life expectancy of these patients, allowing for the development of new clinical features and a more thorough and multimodal evaluation of previously unknown characteristics.

\section{Acknowledgment}

This manuscript does not include any nonauthor contributors to acknowledge.

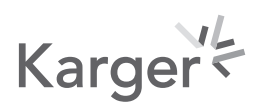




\section{Statement of Ethics}

This study protocol was reviewed, and the need for approval was waived by the Ethics Committee of Centro Hospitalar Universitário de S. João. This study adhered to tenets of the Declaration of Helsinki. The legal guardian of the case gave written informed consent to publish this case (including publication of images).

\section{Conflict of Interest Statement}

One author of this publication (Elisa Leão-Teles) is a member of the European Reference Network for Rare Hereditary Metabolic Disorders (MetabERN) - Project ID No. 739543.

\section{Funding Sources}

None of the authors have received any contribution for this submission.

\section{Author Contributions}

Augusto Magalhães (ophthalmologist) observed and followed the subject of the case report, redacted the manuscript, and contributed to its revision. Rodrigo Vilares-Morgado (ophthalmologist) observed the subject of the case report, redacted the manuscript, and contributed to its revision. Ana Maria Cunha (ophthalmologist) observed the subject of the case report and contributed to the revision of the manuscript. Elisa Leão-Teles (pediatrician) has been following and treating the subject of the case report and contributed to the revision of the manuscript. Manuel Falcão (ophthalmologist) performed optical coherence tomography and other retinal imaging techniques and contributed to the revision of the manuscript. Ângela Carneiro (ophthalmologist) oversaw the redaction of the case report, contributed to the revision process, and approved its final version. Fernando Falcão-Reis (ophthalmologist) oversaw the redaction of the case report, contributed to the revision process, and approved its final version.

\section{Data Availability Statement}

All data generated or analyzed during this study are included in this case report. No other clinical data regarding this patient are available. Further enquiries can be directed to the corresponding author.

\section{References}

1 Kobayashi H. Recent trends in mucopolysaccharidosis research. J Hum Genet. 2019;64(2):127-37.

2 Tomatsu S, Pitz S, Hampel U. Ophthalmological findings in mucopolysaccharidoses. J Clin Med. 2019;8(9):1467.

3 Leong HY, Abdul Azize NA, Chew HB, Keng WT, Thong MK, Mohd Khalid MKN, et al. Clinical, biochemical and genetic profiles of patients with mucopolysaccharidosis type IVA (Morquio A syndrome) in Malaysia: the first national natural history cohort study. Orphanet J Rare Dis. 2019;14(1):143.

4 Montano AM, Tomatsu S, Gottesman GS, Smith M, Orii T. International Morquio A registry: clinical manifestation and natural course of Morquio A disease. J Inherit Metab Dis. 2007;30(2):165-74.

5 Kasmann-Kellner B, Weindler J, Pfau B, Ruprecht KW. Ocular changes in mucopolysaccharidosis IV A (Morquio A syndrome) and long-term results of perforating keratoplasty. Ophthalmologica. 1999;213(3):200-5.

\section{Karger's}


6 Yabe H, Tanaka A, Chinen Y, Kato S, Sawamoto K, Yasuda E, et al. Hematopoietic stem cell transplantation for Morquio A syndrome. Mol Genet Metab. 2016;117(2):84-94.

7 Lachmann RH. Enzyme replacement therapy for lysosomal storage diseases. Curr Opin Pediatr. 2011;23(6): 588-93.

8 Dangel ME, Tsou BH. Retinal involvement in Morquio's syndrome (MPS IV). Ann Ophthalmol. 1985;17(6): 349-54.

9 Ashworth J, Flaherty M, Pitz S, Ramlee A. Assessment and diagnosis of suspected glaucoma in patients with mucopolysaccharidosis. Acta Ophthalmol. 2015;93(2):e111-7.

10 Magalhães A, Meira J, Cunha AM, Moreira RJ, Leão-Teles E, Falcão M, et al. Fundoscopic changes in MaroteauxLamy syndrome. Case Rep Ophthalmol Med. 2019;2019:4692859.

11 Kapoor S, Liasis A, Nischal KK. Choroidal deposits in a patient with mucopolysaccharidoses type 1. JAMA Ophthalmol. 2020;138(8):e194435.

12 Entezari M, Karimi S, Ramezani A, Nikkhah H, Fekri Y, Kheiri B. Choroidal thickness in healthy subjects. J Ophthalmic Vis Res. 2018;13(1):39-43.

13 Kaderli A, Acar MA, Ünlü N, Üney G, Örnek F. The correlation of hyperopia and choroidal thickness, vessel diameter and area. Int Ophthalmol. 2018;38(2):645-53.

14 Özkan B, Koçer ÇA, Altintaș Ö, Karabaş L, Acar AZ, Yüksel N. Choroidal changes observed with enhanced depth imaging optical coherence tomography in patients with mild graves orbitopathy. Eye. 2016;30(7):917-24.

15 Pinheiro-Costa J, Viana Pinto J, Perestrelo S, Beato JN, Torrão L, Brandão E, et al. Increased choroidal thickness in keratoconus patients: perspectives in the disease pathophysiology. J Ophthalmol. 2019;2019:2453931.

16 Iwamoto M, Nawa Y, Maumenee IH, Young-Ramsaran J, Matalon R, Green WR. Ocular histopathology and ultrastructure of Morquio syndrome (systemic mucopolysaccharidosis IV A). Graefes Arch Clin Exp Ophthalmol. 1990;228(4):342. 